\title{
Comorbidities in patients with COPD and pulmonary rehabilitation: do they matter?
}

\author{
Frits M.E. Franssen ${ }^{1}$ and Carolyn L. Rochester ${ }^{2,3}$ \\ Number 4 in the Series "Thematic Review Series on Pulmonary Rehabilitation" \\ Edited by M.A. Spruit and E.M. Clini
}

\begin{abstract}
Affiliations: ${ }^{1}$ Program Development Center, Center of Expertise for Chronic Organ Failure (CIRO+), Horn, The Netherlands. ${ }^{2}$ Section of Pulmonary, Critical Care and Sleep Medicine, Yale University School of Medicine, New Haven, CT, and ${ }^{3}$ A Connecticut Healthcare System, West Haven Campus, West Haven, CT, USA.

Correspondence: F.M.E. Franssen, Program Development Center, Center of Expertise for Chronic Organ Failure (CIRO+), Hornerheide 1, 6085 NM Horn, The Netherlands. E-mail: fritsfranssendaciro-horn.nl

ABSTRACT It is now recognised that chronic obstructive pulmonary disease (COPD) is a heterogeneous disease with many systemic features. Cardiovascular, metabolic, musculoskeletal and psychological comorbidities contribute to the morbidity and mortality in all stages of the disease. The presence of comorbid conditions has important consequences for disease assessment and management. In addition to treatment of the structural and functional changes in the lungs, clinical programmes for COPD should also assess and manage patients' comorbidities. Thus, there is an increasing need to understand the interaction between existing therapies and comorbidities. Pulmonary rehabilitation is an evidence-based intervention that has been shown to improve clinical outcomes in COPD. However, the impact of comorbidities on outcomes of pulmonary rehabilitation and vice versa is only partially understood. To date, there is limited information on the need for adapting specific interventions in pulmonary rehabilitation to comorbidities or the potential adverse effects of pulmonary rehabilitation in these patients. This article addresses the currently available literature and suggests novel areas for research.
\end{abstract}

0

@ERSpublications

Comorbidities in patients with COPD and pulmonary rehabilitation: do they matter? http://ow.ly/qy5MK

\section{Introduction}

Chronic obstructive pulmonary disease (COPD) is characterised by persistent airflow limitation resulting from an enhanced inflammatory response in the lungs [1]. It is a major cause of morbidity and mortality around the world. In 2010, COPD ranked second as a cause of disability-adjusted life years in the USA [2] and it is projected to become the fourth leading cause of death worldwide in 2030 [3]. Although defined by abnormal spirometry, it is well recognised that COPD is more than a respiratory disease. Comorbidities, defined as the coexistence of other medical conditions alongside COPD, contribute to the severity of disease in individual patients [1]. Although not necessarily causally associated with the disease, the existence of COPD

Previous articles in this Series. No. 1: Gloeckl R, Marinov B, Pitta F. Practical recommendations for exercise training in patients with COPD. Eur Respir Rev 2013; 22: 178-186. No. 2: Hill K, Vogiatzis I, Burtin C. The importance of components of pulmonary rehabilitation, other than exercise training, in COPD. Eur Respir Rev 2013 22: 405-413. No. 3: Holland AE, Wadell K, Spruit MA. How to adapt the pulmonary rehabilitation programme to patients with chronic respiratory disease other than COPD. Eur Respir Rev 2013; 22: 577-586.

Received: Oct 052013 | Accepted after revision: Oct 312013

Conflict of interest: None declared.

Provenance: Submitted article, peer reviewed.

Copyright CERS 2014. ERR articles are open access and distributed under the terms of the Creative Commons Attribution Non-Commercial Licence 3.0. 
may actually increase the risk of other diseases [4]. Comorbidities can occur in patients with any degree of airflow limitation and are not restricted to patients with advanced COPD [5]. Moreover, comorbidities are associated with increased morbidity and mortality. The increased understanding of COPD as a systemic disease has important clinical implications. In addition to traditional pharmacological therapy focused on treating chronic airflow limitation, management of COPD now requires a more holistic approach, including the assessment and appropriate treatment of comorbid conditions. Pulmonary rehabilitation is a nonpharmacological intervention with potential benefits for patients with comorbid conditions.

\section{Prevalence of comorbidities in COPD}

Medical comorbidities are common among COPD patients, and occur across the spectrum of disease severity [6]. Multiple comorbidities often coexist and several clusters can be discriminated [7]. In one analysis of the prevalence of comorbidities associated with COPD, 32\% had one additional condition, and $39 \%$ had two or more concurrent medical conditions [8]. In another recent analysis, COPD patients were reported to have a median of nine comorbidities [9]. Comorbidities commonly associated with COPD are listed in table 1 . The prevalence of each type of comorbidity or combined diseases varies somewhat across studies, depending on the patient population evaluated, the methods of patient evaluation used and the definitions of diseases [7]. Cardiovascular disease (including hypertension, atherosclerotic coronary artery disease, congestive heart failure, atrial fibrillation, stroke and peripheral vascular disease) is one of the most common comorbidities. Overall, individuals with COPD have a 2-3-fold increased risk of cardiovascular conditions as compared to age-matched controls when adjusted for cigarette smoking [10, 11]. Endothelial dysfunction and increased arterial stiffness $[12,13]$ begin early in the course of COPD [14] and probably represent the onset of atherosclerotic disease. One large cohort study identified a $40 \%$ prevalence of hypertension among COPD patients [15]. Left ventricular dysfunction, which is commonly unrecognised, affects up to $20 \%$ of COPD patients [16, 17]. Systolic and/or diastolic dysfunction may be present. Pulmonary hypertension is evident in up to $50 \%$ of patients with very severe COPD [18].

Skeletal muscle dysfunction and loss of fat-free mass are other important comorbidities in COPD. Skeletal muscle dysfunction in COPD is characterised by alterations in fibre type, capillarisation, reduced oxidative enzyme capacity and altered energy metabolism [19]. Loss of fat-free mass can occur in up to 10-15\% of persons with mild-to-moderate COPD and up to $50 \%$ of persons with severe disease [20,21]. It is prevalent among persons who are underweight and among obese persons with COPD [22]. Skeletal muscle dysfunction is associated with reduced strength, endurance and exercise capacity, as well as worse quality of life, increased risk of hospitalisation and mortality [19, 23, 24]. Importantly, several features of skeletal muscle dysfunction can be stabilised or reversed by undertaking pulmonary rehabilitation.

Additionally, up to $50 \%$ of persons with COPD have osteoporosis $[25,26]$; the prevalence is highest among persons with more severe airflow obstruction. Hip and vertebral compression fractures are common

\section{TABLE 1 Comorbidities commonly associated with chronic obstructive pulmonary disease}

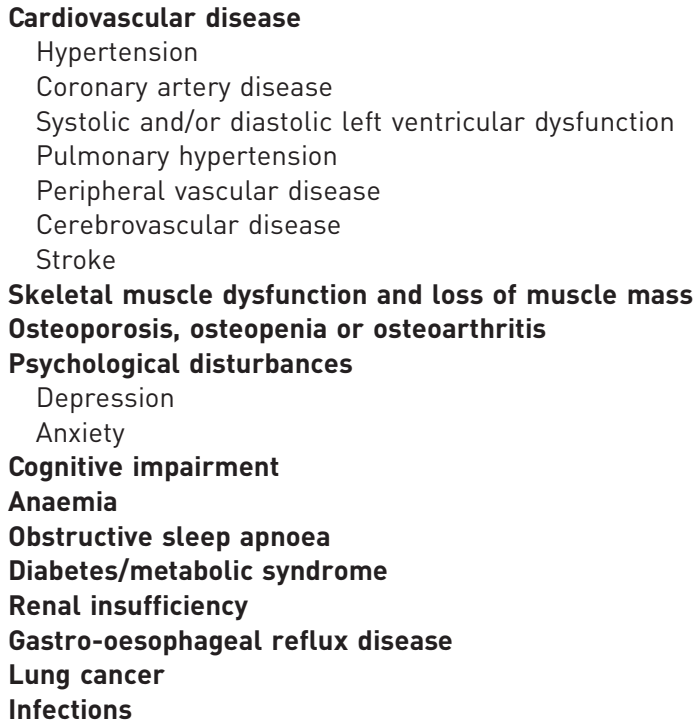


sequelae of osteoporosis, with potential major negative impact on a patients' functional status. Pain and kyphosis related to vertebral compression fractures can alter mechanics of breathing and precipitate decompensation in gas exchange or overt respiratory failure.

Depression affects up to $42 \%$ of patients with COPD [27]; the prevalence increases to $60 \%$ among persons who are dependent on supplemental oxygen [28]. Cognitive impairment has also been reported in $77 \%$ of COPD patients with hypoxaemia [29], and is often also evident among some non-hypoxaemic patients [30]. Additionally, 10-19\% of COPD patients are afflicted with anxiety.

Normochromic, normocytic anaemia is another common feature of COPD [31]. Anaemia is present across the spectrum of COPD severity, with an estimated prevalence of 10-30\% [32-34]. Obstructive sleep apnoea (OSA) is present among an estimated $10 \%$ of COPD patients, and up to $20 \%$ of patients with OSA have concurrent COPD [35]. The concurrence of COPD and OSA is termed "the overlap syndrome". Diabetes is present in $\sim 12 \%$ of COPD patients [36], while the frequency of glucose intolerance is much higher [7] and renal insufficiency may be present in 20-22\% [37]. Gastro-oesophageal reflux disease affects up to $60 \%$ of COPD patients $[38,39]$ and is associated with an increased risk of exacerbations $[40,41]$. Finally, although the overall prevalence is uncertain, lung cancer [42] and respiratory infections [43] are additional common comorbidities with major impact on patient symptoms and outcomes.

\section{Impact of comorbidities on outcomes in COPD}

Comorbid conditions contribute to the societal and economic burden of COPD. The number of comorbidities is related to the total annual costs of the disease [44]. In addition to age and the presence of chronic symptoms, comorbidities are the most predictive factors of future healthcare costs [45]. The presence of comorbidities is also associated with increased risk of hospitalisation [15]. In addition to respiratory complaints, the incidence of hospitalisations for non-respiratory causes, including cardiovascular, gastro-intestinal and musculoskeletal diseases, is significantly increased in patients with COPD compared to controls [45]. Importantly, the presence and number of comorbidities has a negative impact on several patient-related outcomes. Even after accounting for differences in age, sex and smoking history, COPD patients with comorbidities, in particular cardiovascular disease and diabetes, are generally more dyspnoeic and have a reduced exercise capacity in comparison to patients without comorbidities [46]. Similar observations have been reported for the presence of psychological disorders and anaemia in COPD. Among patients with moderate-to-severe pulmonary emphysema, the presence of anxiety is associated with worse shortness of breath, health status and exercise performance [47]. Patients with comorbid depression reported worse health status and functional impairment, while treatment adherence was reduced [48]. Anaemia is also associated with worse dyspnoea, reduced exercise capacity and worse quality of life [49]. In addition, a higher number of self-reported comorbidities are associated with increased dyspnoea [9]. The presence of three or more comorbid diseases in COPD is more predictive of decreased health status than any demographic or clinical variable [50]. Another study indicated that the influence of comorbidity on health status was particularly seen in patients with mild-to-moderate disease, while in patients with severe airflow limitation, the impact of comorbid disease was less pronounced [51]. Finally, comorbidities are associated with increased mortality risk in COPD [15, 46, 52-55]. Given the negative impact of comorbidities on patient symptoms, disability, health status, hospitalisation risk, healthcare costs and mortality, interventions that can stabilise or reduce the severity of comorbid conditions are needed. Pulmonary rehabilitation is one intervention which, by virtue of its exercise training, emphasis on selfmanagement and behaviour change, and psychosocial support, may achieve this goal in conjunction with optimised pharmacological therapies.

\section{Impact of comorbidities on outcomes of pulmonary rehabilitation}

Pulmonary rehabilitation is a comprehensive intervention to improve the physical and psychological functioning of patients with COPD and promote the long-term adherence to health-enhancing behaviours [56]. Pulmonary rehabilitation has been demonstrated clearly to reduce dyspnoea, increase exercise capacity and improve quality of life in these patients [56], and is indicated for persons who remain symptomatic despite optimal pharmacological therapy. Exercise training, nutritional support, education and behaviour change are therapies frequently included in pulmonary rehabilitation programmes [56]. The intervention is based on an assessment of the integrated health status of the individual patient, and addresses and treats systemic effects and comorbidities of the disease [57]. Although the complex and heterogeneous nature of COPD is now more fully understood, to date, few studies have addressed the impact of comorbidities on outcomes of pulmonary rehabilitation. These studies are summarised in table 2.

In an observational retrospective cohort study, CRISAFULLI et al. [59] studied the prevalence and impact of comorbidities on pulmonary rehabilitation outcomes in 2962 patients with moderate-to-severe COPD 
TABLE 2 Overview of the impact of comorbidities on pulmonary rehabilitation outcomes in chronic obstructive pulmonary disease

Study Patients n Design Conclusion

$\begin{array}{cc}\text { CARREIRO [58] } & 114 \quad \text { Retrospective, outpatient pulmonary } \\ \text { rehabilitation }\end{array}$

CRISAfULLI [59]

CRisafulli [60]

316

GARROD [61]

GREENING [62]

VON LEUPOLDT [63]

VAGAGGINI [65]

60

WALSH [66]

203

Young [67]
Retrospective, outpatient pulmonary rehabilitation

Retrospective, outpatient pulmonary

rehabilitation

Retrospective, out- and inpatient pulmonary rehabilitation

Prospective, outpatient pulmonary rehabilitation

Prospective, outpatient pulmonary rehabilitation

Retrospective, outpatient pulmonary rehabilitation

Prospective, outpatient pulmonary rehabilitation
Retrospective, outpatient pulmonary rehabilitation

Prospective, outpatient pulmonary rehabilitation
Ischaemic heart disease negatively influenced improvement in health status

Psychological symptoms predicted lower improvement in symptoms

Metabolic diseases were inversely related to improvement in 6MWD

Cardiac diseases were inversely related to improvement in health status

Osteoporosis was inversely related to improvement in $6 \mathrm{MWD}$

Depressed patients were found to be considerably more at risk of drop-out than nondepressed patients

No difference was observed in improvements in exercise performance and health status between BMI categories

No effect of anxiety on 6MWD after pulmonary rehabilitation

Depression was associated with reduced improvement in dyspnoea during activities

No difference was observed in improvement in 6MWD, health status, unsupported arm lifts and functional performance between obese and nonobese patients

Cardiovascular disease was related to lack of improvement in 6MWD and health status, being overweight was associated with more pronounced improvement in 6MWD

Metabolic disease was associated with better improvement in 6MWD

Nonadherent patients were no more likely to have psychological symptoms than adherent patients

6MWD: 6-min walk distance; BMI: body mass index.

treated in a comprehensive programme. The programme consisted of a minimum of 15 daily sessions of endurance and strength training, education and behavioural, nutritional and psychosocial counselling. The presence of comorbidities was documented based on a review of patients' medical records and were counted as single comorbidities and combined diseases: heart disease (24\%), metabolic disease (62\%), skeletal disease $(7 \%)$ and other disease $(8 \%)$. Overall, $51 \%$ of the patients had at least one comorbidity. The percentage of patients with a clinically meaningful improvement in dyspnoea or health status after pulmonary rehabilitation varied between comorbidity categories, while changes in functional exercise performance were comparable. Compared to patients with without or with one comorbidity, patients with two or more comorbidities had changes in the Medical Research Council scale for dyspnoea that exceeded the minimal clinically important difference (MCID) more frequently, although they reached the MCID for St George's Respiratory Questionnaire (SGRQ), a measure for quality of life, less often. In multiple logistic regression analysis, the presence of metabolic disease and/or heart disease was inversely related to improvements in functional exercise performance (assessed by 6-min walk distance (6MWD)) and SGRQ [59]. Moreover, the overall impact of comorbidities, assessed by the Charlson Index [68], inversely predicted outcomes after pulmonary rehabilitation. The results of this study suggested that patients with multiple comorbidities, especially those with metabolic disease and heart disease, might be less likely to benefit or might achieve lower degrees of improvement in exercise capacity or quality of life from participation in pulmonary rehabilitation.

In another retrospective study including 60 patients with moderate-to-severe COPD who completed an outpatient exercise training programme, all patients achieved significant improvements in 6MWD and decreases in SGRQ, except for the patients with cardiovascular comorbidities [60]. Being overweight was an independent predictor of efficacy of pulmonary rehabilitation and it was hypothesised that overweight 
patients may be more deconditioned and so they may benefit more from exercise training [65]. However, this was not confirmed in other studies. RAMACHANDRAN et al. [64] investigated the impact of obesity on pulmonary rehabilitation outcomes. In their retrospective analysis, obese patients had poorer functional performance and health status compared to nonobese patients at baseline, despite a lower degree of airflow limitation. However, improvements in $6 \mathrm{MWD}$, health status (assessed with the chronic respiratory questionnaire) and functional performance (assessed with the Pulmonary Functional Status Scale) following outpatient pulmonary rehabilitation were similar between obese and nonobese COPD patients [64]. GREENING et al. [62] also investigated the impact of obesity on pulmonary rehabilitation outcomes using a database of 601 patients attending a 7-week pulmonary rehabilitation programme consisting of walkingbased physical training and education. While exercise performance was more impaired at baseline in obese patients, the programme was comparably effective in increasing exercise capacity and health status in obese compared to nonobese patients [62]. In another study [61], body mass index was not related to outcomes after pulmonary rehabilitation in patients with COPD.

A subsequent prospective study by CRISAFulli et al. [60] included 316 patients with moderate-to-severe COPD attending an outpatient pulmonary rehabilitation programme, consisting of peripheral limb training, education, chest physiotherapy and psychological and nutritional counselling three times a week with a minimum of 21 sessions. $62 \%$ of patients had at least one associated chronic condition. Self-reported hypertension, dyslipidaemia, diabetes, coronary disease, chronic heart failure and osteoporosis were the six most prevalent comorbidities, while the frequency distribution of the combined diseases was comparable to their previous study [59]. There was no difference in withdrawal rates from pulmonary rehabilitation between comorbidity categories. Although the coexistence of osteoporosis negatively predicted 6MWD improvement, unlike the findings of the authors' prior retrospective analysis, no other comorbidities were related to poorer outcomes after pulmonary rehabilitation. Also, the number of comorbidities was not predictive of outcomes. WALSH et al. [66] retrospectively studied the predictors of response to pulmonary rehabilitation, including the combined comorbidities as previously proposed [59]. Pulmonary rehabilitation consisted of education and exercise training twice a week for 7 weeks. Response was defined as a clinically important improvement in 6MWD. In 203 participants with moderate-to-severe COPD, data on comorbidities were retrieved from the medical records and classified as the presence of musculoskeletal (osteoporosis and arthrosis), cardiac (ischaemic heart disease, chronic heart failure and cardiac arrhythmias) and metabolic (systemic hypertension, diabetes and dyslipidaemia) diseases. In addition to younger age, the presence of metabolic disease was an independent predictor of a better response in exercise capacity after pulmonary rehabilitation [66]. Similar to the prospective study by CRISAFULLI et al. [60], the other categories of comorbidities and the number of comorbid conditions were not related to pulmonary rehabilitation outcomes.

Additionally, CARREIRO et al. [58] performed a retrospective study including 114 patients with mostly severe COPD that attended an 8-week pulmonary rehabilitation programme. The intervention consisted of disease self-management, education and modification of risk factors, along with psychosocial and nutritional support, breathing techniques and exercise training. Comorbidities were recorded based on the patients' diagnostic and medical data and prescriptions available on their clinical records. Co-occurring chronic medical conditions were grouped as: metabolic (71.1\%), cardiovascular (67.5\%), respiratory (59.9\%), psychological (21.1\%), osteoarticular (10.5\%) and other comorbidities (59.6\%). 96\% of patients had one or more comorbidity and more than half of the patients improved beyond the MCID for functional exercise capacity, health status or dyspnoea. Although there was no association between the number of comorbidities $(0,1$ or $>1)$ and pulmonary rehabilitation outcomes, the presence of ischaemic heart disease negatively influenced the improvement in health status. Also, anxiety/depression predicted lower improvement in dyspnoea after pulmonary rehabilitation. Drop-out rates were almost $18 \%$, but there were no differences in comorbidities between patients who completed the pulmonary rehabilitation and those who dropped out. In another study, VON LEUPOLDT et al. [63] studied the impact of anxiety and depression on pulmonary rehabilitation outcomes. The study included 238 patients with COPD who completed a standardised, 3-week, multidisciplinary outpatient pulmonary rehabilitation programme performed for 6 hours per day for 5 days per week. The presence of anxiety and depression was independently associated with increased dyspnoea, reduced quality of life and reduced 6MWD before pulmonary rehabilitation [63]. Although psychological symptoms improved after pulmonary rehabilitation, anxiety and depression were related to poor outcomes after pulmonary rehabilitation. Depressed patients were found to be at a considerably higher risk of drop-out than nondepressed patients [61]. However, Young et al. [67] also studied predictors of nonadherence to pulmonary rehabilitation programmes. They did not observe more anxiety or depression in the group of COPD patients that did not complete a 4 week, hospital-based outpatient pulmonary rehabilitation programme compared to patients that were adherent. 
In summary, medical comorbidities are extremely common among patients with COPD referred to pulmonary rehabilitation programmes. Although several studies have investigated the impact of comorbidities on pulmonary rehabilitation outcomes in COPD, at present, the results are inconclusive. Although some retrospective studies reported worse outcomes in patients with cardiovascular, metabolic or psychological comorbid conditions, others, including one prospective study, did not confirm this or reported opposite effects. Available studies suggest, however, that the presence of specific conditions or combinations of conditions, rather than the total number of comorbidities, may impact on programme adherence and selected outcomes of pulmonary rehabilitation. This is an area for future additional study.

\section{Adapting pulmonary rehabilitation programmes for patients with comorbidities}

The studies discussed in the previous section investigated the effects of general pulmonary rehabilitation programmes in patients with COPD and comorbidities. However, the effects of specific pulmonary rehabilitation programmes adapted for patients with co-occurring medical conditions remain largely unknown. Programmes specifically developed for these patients might reduce any potential adverse impact of comorbidity on outcomes and adherence. Also, they might have a positive effect on the course of the comorbidity and thus contribute to improved integrated health status. For example, McNAMARA et al. [69] performed a prospective randomised controlled trial comparing the effects of water-based exercise training, land-based exercise training or control in COPD patients with musculoskeletal or neurological comorbidities or obesity. Pulmonary rehabilitation consisted of three weekly outpatient sessions of $1 \mathrm{~h}$ for 8 weeks. Exercise in water and on land was matched as closely as possible for intensity, duration and muscle groups trained [69]. Drop-out rates were low and comparable between interventions. Compared to land-based exercise training, water-based exercise training significantly increased incremental and endurance shuttle walk distance and improved the fatigue domain of the chronic respiratory questionnaire. This suggested that water-based training is more effective in COPD patients with physical comorbidities. Additional research is needed to identify the optimal programme modifications likely to lead to the greatest gains in various outcomes following pulmonary rehabilitation for patients with differing comorbidities.

\section{Effects of pulmonary rehabilitation on comorbidities}

Physical activity and regular exercise are both recommended and are beneficial not only for patients with COPD, but also for patients with cardiovascular disease, musculoskeletal disease, obesity, diabetes mellitus and most other chronic medical conditions [70-74]. In fact, there is a striking similarity between the peripheral muscle dysfunction and structural and metabolic abnormalities evident in patients with COPD and those found in other chronic organ diseases [75]. It is well established that the structural and functional changes in skeletal muscle in COPD can be stabilised or reversed with the pulmonary rehabilitation intervention $[19,23,56]$. In patients with heart failure or type 2 diabetes mellitus, prescribed exercise, involving aerobic and resistance training modalities, is also evidence-based treatment [76, 77]. Thus, exercise training in the context of pulmonary rehabilitation is important for individuals with COPD and comorbidities, as it has positive effects on both.

Although almost $40 \%$ of COPD patients entering pulmonary rehabilitation fulfil diagnostic criteria for metabolic syndrome [78], defined as a complex of risk factors for cardiovascular disease, the effects of pulmonary rehabilitation on abdominal obesity, dyslipidaemia, increased blood pressure and insulin sensitivity currently remain unknown. VIVODTZEV et al. [79] were the first to show significant improvements in peripheral pulse wave velocity (PWV), an independent predictor of cardiovascular events and mortality [80], in seven patients with COPD who underwent a 4-week aerobic endurance training programme compared to 10 non-exercising case-control subjects. In line with this, GALE et al. [81] showed a small, but significant, reduction in aortic PWV in an observational study of 32 patients with COPD following an outpatient-based three weekly pulmonary rehabilitation programme including endurance training and resistance training. However, the change in aortic PWV following pulmonary rehabilitation was highly variable. The overall impact of participation in pulmonary rehabilitation on the risk for developing cardiovascular disease or on cardiovascular disease-related outcomes among patients with COPD remains unknown. This represents an important area for future study.

In the landmark study by GRIFFITHS et al. [82], a 6-week outpatient multidisciplinary pulmonary rehabilitation programme in patients with severe COPD resulted in significant decreases in hospital anxiety and depression scores, in comparison with standard medical management. In a study with a comparable COPD population, a 16-week tertiary care outpatient pulmonary rehabilitation programme without specific psychological interventions resulted in decreased psychosocial morbidity, compared to usual care [83]. A systematic review and meta-analysis performed to estimate the clinical effect of pulmonary rehabilitation (with or without education) on anxiety and depression in COPD [84] included six randomised controlled trials comparing pulmonary rehabilitation with standard care. When compared with standard care, 
comprehensive pulmonary rehabilitation, including exercise training, education and psychosocial support, was significantly more effective in reducing anxiety and depression in the short term. Exercise training alone was not associated with significant reductions in either anxiety or depression when compared with standard care [84].

\section{Special considerations for patients with comorbidities entering pulmonary rehabilitation}

Knowledge of medical comorbidities raises several important practical considerations for the assessment and management of patients with COPD who are entering pulmonary rehabilitation programmes. Although no formal guidelines exist to direct a standard approach to diagnosis and assessment of comorbidities in COPD patients, recognition of comorbidities is essential because treating the comorbidities can have a beneficial effect on COPD and vice versa. Early intervention may potentially influence the course and prognosis of the disease, and recognising comorbidities in the context of choosing patients for and monitoring patients within pulmonary rehabilitation programmes is necessary to ensure patients' safety. The pre-rehabilitation medical history and physical examination should include detailed questions regarding symptoms and signs of the common comorbidities associated with COPD. It should not be assumed that other healthcare providers will always have considered these issues prior to patient referral to pulmonary rehabilitation. A baseline electrocardiogram is recommended [85]; echocardiography, cardiac stress testing (including pharmacological or exercise-based stress testing) and measurement of circulating natriuretic peptide levels [76] may be advisable for persons with exertional dizziness or chest pain, peripheral oedema, or whose dyspnoea is out of proportion to the severity of airflow obstruction. Patients should be asked about symptoms of leg fatigue and claudication. Results of recent complete blood counts and chemistries, bone density testing, as well as any available assessments of cognitive function, psychological well-being or sleep disturbances, should be reviewed. Balance and fall risk should be assessed. Consultation with other specialists, including cardiologists, rheumatologists, orthopaedists or physiatrists may be needed to determine safe exercise parameters. Cardiopulmonary exercise testing may be helpful to identify the principal mechanisms of exercise impairment and guide a safe exercise training programme among persons with multiple potential factors contributing to their activity intolerance. The medical director and/or pulmonary rehabilitation programme coordinator should communicate closely with the referring healthcare provider to suggest additional diagnostic testing prior to pulmonary rehabilitation where needed. Screening questionnaires for anxiety, depression and/or cognitive impairment are often administered during the pulmonary rehabilitation baseline patient assessment [86]. Special equipment needs must be considered. Realistic training targets must be formulated that meet individuals' goals. For persons with cardiac disease, there are some suggested contraindications to exercise that should be considered (table 3) [87]. Telemetry monitoring should be considered for persons with a history of serious arrhythmias or prior sudden cardiac death, severe pulmonary hypertension or left ventricular dysfunction, coronary artery disease with exercise-induced ischaemia, implanted defibrillator devices, abnormal heart rate response to exercise or inability to self-manage exercise intensity [88]. Pulmonary rehabilitation staff must be trained to recognise the symptoms and signs of the common comorbidities, since some comorbidities may not have been recognised prior to the patient's enrolment in pulmonary rehabilitation. Pulmonary rehabilitation care providers may also recommend selected interventions (e.g. nutritional counselling, mental health care, cognitive testing, etc.) based on their observations of the patient during pulmonary rehabilitation. Pulmonary rehabilitation staff should also be able to provide advice to patients regarding aspects of their management, recognise features of disease destabilisation (such as unstable angina or cardiac arrhythmia, transient ischaemic attack, etc.) and notify the referring healthcare provider and/or refer patients for additional specialty care when needed. Patient education sessions should also address the long-term management of common comorbidities. Ideally, targeted goals should be established and education and monitoring should be provided with regard to weight management, diabetes, blood pressure, lipid management, psychological disturbances, bone and muscle health, sleep disordered breathing and smoking cessation, in addition to topics regarding COPD typically included in pulmonary rehabilitation programmes.

\section{Future perspectives}

Although there is a wealth of studies indicating the beneficial effects of pulmonary rehabilitation in patients with COPD, including those with comorbidities, there are important limitations to the currently available literature. In addition to being descriptive, previous studies evaluating the impact of comorbidities on pulmonary rehabilitation outcomes have used data retrieved from medical records, without objective confirmation of the diagnoses or quantification of their severity. However, since comorbidities in COPD are often undiagnosed and untreated [89], they may blunt the effects of pulmonary rehabilitation. Therefore, objectively identifying these comorbidities in patients referred for pulmonary rehabilitation is an important first step [7]. Also, there are as yet no prospective studies directly comparing the effects of pulmonary 


\section{TABLE 3 Contraindications to exercise training in patients with chronic heart failure}

\section{Relative contraindications}

$\geqslant 1.8 \mathrm{~kg}$ increase in body mass over previous $1-3$ days

Concurrent continuous or intermittent dobutamine therapy

Decrease in systolic blood pressure with exercise

New York Heart Association functional class IV

Complex ventricular arrhythmias at rest or appearing with exertion

Supine resting heart rate $\geqslant 100$ beats $\cdot \mathrm{min}^{-1}$

Pre-existing comorbidities

\section{Absolute contraindications}

Progressive worsening of exercise tolerance, or dyspnoea at rest or on exertion over previous 3-5 days

Significant ischaemia at low work rates $(<2$ METS or $\cong 50 \mathrm{~W}$ )

Uncontrolled diabetes

Acute systemic illness or fever

Recent embolism

Thrombophlebitis

Active pericarditis or myocarditis

Moderate-to-severe aortic stenosis

Regurgitant valvular heart disease requiring surgery

Myocardial infarction within previous 3 weeks

New onset atrial fibrillation

METS: metabolic equivalents. Reproduced from [87].

rehabilitation in COPD patients with and without single or combined comorbidities. Furthermore, studies in which pulmonary rehabilitation programmes were adapted in order to address or improve specific comorbid conditions or in which comorbidity was the primary outcome are lacking. Also, the long-term effects of pulmonary rehabilitation on the development and natural course of comorbidities in COPD have not been studied.

\section{Conclusions}

Medical comorbidities are common among patients referred for pulmonary rehabilitation. The impact of comorbidities on the outcomes of pulmonary rehabilitation in patients with COPD remains uncertain. Although current evidence suggests that the overall number of comorbidities does not affect adherence and outcomes of pulmonary rehabilitation, contradicting results have been published regarding the impact of cardiovascular, metabolic and psychological comorbid conditions on selected pulmonary rehabilitation outcomes. Little is known about the potential benefits of pulmonary rehabilitation on the development and clinical course of comorbidities. The aim for the future is to optimise the design of clinical trials in order to address specific comorbidities during pulmonary rehabilitation and move towards a tailored approach for COPD patients with comorbidities.

\section{Acknowledgements}

We thank E.M. Clini (Dept of Medical and Surgical Sciences, University of Modena and Reggio Emilia, Modena, Italy) for his critical evaluation of this review.

\section{References}

1 Vestbo J, Hurd SS, Agustí AG, et al. Global strategy for the diagnosis, management, and prevention of chronic obstructive pulmonary disease: GOLD executive summary. Am J Respir Crit Care Med 2013; 187: 347-365.

Murray CJ, Lopez AD. Measuring the global burden of disease. N Engl J Med 2013; 369: 448-457.

Mathers CD, Loncar D. Projections of global mortality and burden of disease from 2002 to 2030. PLoS Med 2006; 3: e442.

Houghton AM. Mechanistic links between COPD and lung cancer. Nat Rev Cancer 2013; 13: 233-245.

Agusti A, Calverley PM, Celli B, et al. Characterisation of COPD heterogeneity in the ECLIPSE cohort. Respir Res 2010; 11: 122.

Barnes PJ, Celli BR. Systemic manifestations and comorbidities of COPD. Eur Respir J 2009; 33: 1165-1185.

7 Vanfleteren LE, Spruit MA, Groenen M, et al. Clusters of comorbidities based on validated objective measurements and systemic inflammation in patients with chronic obstructive pulmonary disease. Am J Respir Crit Care Med 2013; 187: 728-735.

8 Schneider KM, O’Donnell BE, Dean D. Prevalence of multiple chronic conditions in the United States' Medicare population. Health Qual Life Outcomes 2009; 7: 82.

9 Barr RG, Celli BR, Mannino DM, et al. Comorbidities, patient knowledge, and disease management in a national sample of patients with COPD. Am J Med 2009; 122: 348-355.

10 Sin DD. Is COPD really a cardiovascular disease? Chest 2009; 136: 329-330. 
11 Finkelstein J, Cha E, Scharf SM. Chronic obstructive pulmonary disease as an independent risk factor for cardiovascular morbidity. Int J Chron Obstruct Pulmon Dis 2009; 4: 337-349.

12 McAllister DA, Maclay JD, Mills NL, et al. Arterial stiffness is independently associated with emphysema severity in patients with chronic obstructive pulmonary disease. Am J Respir Crit Care Med 2007; 176: 1208-1214.

13 Sabit R, Bolton CE, Edwards PH, et al. Arterial stiffness and osteoporosis in chronic obstructive pulmonary disease. Am J Respir Crit Care Med 2007; 175: 1259-1265.

14 Iwamoto $\mathrm{H}$, Yokoyama A, Kitahara $\mathrm{Y}$, et al. Airflow limitation in smokers is associated with subclinical atherosclerosis. Am J Respir Crit Care Med 2009; 179: 35-40.

15 Mannino DM, Thorn D, Swensen A, et al. Prevalence and outcomes of diabetes, hypertension and cardiovascular disease in COPD. Eur Respir J 2008; 32: 962-969.

16 Rutten FH, Moons KG, Cramer MJ, et al. Recognising heart failure in elderly patients with stable chronic obstructive pulmonary disease in primary care: cross sectional diagnostic study. BMJ 2005; 331: 1379.

17 Rutten FH, Vonken EJ, Cramer MJ, et al. Cardiovascular magnetic resonance imaging to identify left-sided chronic heart failure in stable patients with chronic obstructive pulmonary disease. Am Heart J 2008; 156: 506-512.

18 Thabut G, Dauriat G, Stern JB, et al. Pulmonary hemodynamics in advanced COPD candidates for lung volume reduction surgery or lung transplantation. Chest 2005; 127: 1531-1536.

19 Maltais F, LeBlanc P, Jobin J, et al. Peripheral muscle dysfunction in chronic obstructive pulmonary disease. Clin Chest Med 2000; 21: 665-677.

20 Agusti A, Soriano JB. COPD as a systemic disease. COPD 2008; 5: 133-138.

21 Schols AM, Soeters PB, Dingemans AM, et al. Prevalence and characteristics of nutritional depletion in patients with stable COPD eligible for pulmonary rehabilitation. Am Rev Respir Dis 1993; 147: 1151-1156.

22 Rutten EP, Breyer MK, Spruit MA, et al. Abdominal fat mass contributes to the systemic inflammation in chronic obstructive pulmonary disease. Clin Nutr 2010; 29: 756-760.

23 Skeletal muscle dysfunction in chronic obstructive pulmonary disease. A statement of the American Thoracic Society and European Respiratory Society. Am J Respir Crit Care Med 1999; 159: S1-S40.

24 Maltais F, LeBlanc $\mathrm{P}$, Whittom F, et al. Oxidative enzyme activities of the vastus lateralis muscle and the functional status in patients with COPD. Thorax 2000; 55: 848-853.

25 Jørgensen NR, Schwarz P, Holme I, et al. The prevalence of osteoporosis in patients with chronic obstructive pulmonary disease: a cross sectional study. Respir Med 2007; 101: 177-185.

26 Graat-Verboom L, Wouters EF, Smeenk FW, et al. Current status of research on osteoporosis in COPD: a systematic review. Eur Respir J 2009; 34: 209-218.

27 Maurer J, Rebbapragada V, Borson S, et al. Anxiety and depression in COPD: current understanding, unanswered questions, and research needs. Chest 2008; 134: Suppl. 4, 43S-56S.

28 Lacasse Y, Rousseau L, Maltais F. Prevalence of depressive symptoms and depression in patients with severe oxygen-dependent chronic obstructive pulmonary disease. J Cardiopulm Rehabil 2001; 21: 80-86.

29 Grant I, Heaton RK, McSweeny AJ, et al. Neuropsychologic findings in hypoxemic chronic obstructive pulmonary disease. Arch Intern Med 1982; 142: 1470-1476.

30 Liesker JJ, Postma DS, Beukema RJ, et al. Cognitive performance in patients with COPD. Respir Med 2004; 98: 351-356.

31 Rutten EP, Franssen FM, Spruit MA, et al. Anemia is associated with bone mineral density in chronic obstructive pulmonary disease. COPD 2013; 10: 286-292.

32 John M, Hoernig S, Doehner W, et al. Anemia and inflammation in COPD. Chest 2005; 127: 825-829.

33 Martinez-Rivera C, Portillo K, Muñoz-Ferrer A, et al. Anemia is a mortality predictor in hospitalized patients for COPD exacerbation. COPD 2012; 9: 243-250.

34 John M, Lange A, Hoernig S, et al. Prevalence of anemia in chronic obstructive pulmonary disease: comparison to other chronic diseases. Int J Cardiol 2006; 111: 365-370.

35 Weitzenblum E, Chaouat A. Sleep and chronic obstructive pulmonary disease. Sleep Med Rev 2004; 8: 281-294.

36 Spranger J, Kroke A, Möhlig M, et al. Inflammatory cytokines and the risk to develop type 2 diabetes: results of the prospective population-based European Prospective Investigation into Cancer and Nutrition (EPIC)-Potsdam Study. Diabetes 2003; 52: 812-817.

37 Incalzi RA, Corsonello A, Pedone C, et al. Chronic renal failure: a neglected comorbidity of COPD. Chest 2010; 137: 831-837.

38 Casanova C, Baudet JS, del Valle Velasco M, et al. Increased gastro-oesophageal reflux disease in patients with severe COPD. Eur Respir J 2004; 23: 841-845.

39 Kempainen RR, Savik K, Whelan TP, et al. High prevalence of proximal and distal gastroesophageal reflux disease in advanced COPD. Chest 2007; 131: 1666-1671.

40 Rascon-Aguilar IE, Pamer M, Wludyka P, et al. Role of gastroesophageal reflux symptoms in exacerbations of COPD. Chest 2006; 130: 1096-1101.

41 Hurst JR, Vestbo J, Anzueto A, et al. Susceptibility to exacerbation in chronic obstructive pulmonary disease. N Engl J Med 2010; 363: 1128-1138.

42 Powell HA, Iyen-Omofoman B, Baldwin DR, et al. Chronic obstructive pulmonary disease and risk of lung cancer: the importance of smoking and timing of diagnosis. J Thorac Oncol 2013; 8: 6-11.

43 Sethi S. Infection as a comorbidity of COPD. Eur Respir J 2010; 35: 1209-1215.

44 Mapel DW, Hurley JS, Frost FJ, et al. Health care utilization in chronic obstructive pulmonary disease. A casecontrol study in a health maintenance organization. Arch Int Med 2000; 160: 2653-2658.

45 Mannino DM, Watt G, Hole D, et al. The natural history of chronic obstructive pulmonary disease. Eur Respir J 2006; 27: 627-643.

46 Miller J, Edwards LD, Agustí A, et al. Comorbidity, systemic inflammation and outcomes in the ECLIPSE cohort. Respir Med 2013; 107: 1376-1384.

47 Giardino ND, Curtis JL, Andrei AC, et al. Anxiety is associated with diminished exercise performance and quality of life in severe emphysema: a cross-sectional study. Respir Res 2010; 11: 29.

48 Norwood R, Balkissoon R. Current perspectives on management of co-morbid depression in COPD. COPD 2005; 2: $185-193$.

49 Cote C, Zilberberg MD, Mody SH, et al. Haemoglobin level and its clinical impact in a cohort of patients with COPD. Eur Respir J 2007; 29: 923-929. 
an Manen JG, Bindels PJ, Dekker EW, et al. Added value of co-morbidity in predicting health-related quality of life in COPD patients. Respir Med 2001; 95: 496-504.

51 Ferrer M, Alonso J, Morera J, et al. Chronic obstructive pulmonary disease stage and health-related quality of life. The Quality of Life of Chronic Obstructive Pulmonary Disease Study Group. Ann Intern Med 1997; 127: 1072-1079.

52 Divo M, Cote C, de Torres JP, et al. Comorbidities and risk of mortality in patients with chronic obstructive pulmonary disease. Am J Respir Crit Care Med 2012; 186: 155-161.

53 Lavie P, Herer P, Lavie L. Mortality risk factors in sleep apnoea: a matched case-control study. J Sleep Res 2007; 16: $128-134$.

54 Lee R, McNicholas WT. Obstructive sleep apnea in chronic obstructive pulmonary disease patients. Curr Opin Pulm Med 2011; 17: 79-83.

55 Schols AM, Broekhuizen R, Weling-Scheepers CA, et al. Body composition and mortality in chronic obstructive pulmonary disease. Am J Clin Nutr 2005; 82: 53-59.

56 Spruit MA, Singh SJ, Garvey C, et al. An official American Thoracic Society/European Respiratory Society Statement: key concepts and advances in pulmonary rehabilitation. Am J Respir Crit Care Med 2013; 188 : e13-e64.

57 Nici L, Zuwallack R. Scope, background and definition of pulmonary rehabilitation. Eur J Phys Rehabil Med 2011; 47: 465-474.

58 Carreiro A, Santos J, Rodrigues F. [Impact of comorbidities in pulmonary rehabilitation outcomes in patients with chronic obstructive pulmonary disease]. Rev Port Pneumol 2013; 19: 106-113.

59 Crisafulli E, Costi S, Luppi F, et al. Role of comorbidities in a cohort of patients with COPD undergoing pulmonary rehabilitation. Thorax 2008; 63: 487-492.

60 Crisafulli E, Gorgone P, Vagaggini B, et al. Efficacy of standard rehabilitation in COPD outpatients with comorbidities. Eur Respir J 2010; 36: 1042-1048.

61 Garrod R, Marshall J, Barley E, et al. Predictors of success and failure in pulmonary rehabilitation. Eur Respir J 2006; 27: 788-794

62 Greening NJ, Evans RA, Williams JE, et al. Does body mass index influence the outcomes of a Waking-based pulmonary rehabilitation programme in COPD? Chron Respir Dis 2012; 9: 99-106.

63 von Leupoldt A, Taube K, Lehmann K, et al. The impact of anxiety and depression on outcomes of pulmonary rehabilitation in patients with COPD. Chest 2011; 140: 730-736.

64 Ramachandran K, McCusker C, Connors M, et al. The influence of obesity on pulmonary rehabilitation outcomes in patients with COPD. Chron Respir Dis 2008; 5: 205-209.

65 Vagaggini B, Costa F, Antonelli S, et al. Clinical predictors of the efficacy of a pulmonary rehabilitation programme in patients with COPD. Respir Med 2009; 103: 1224-1230.

66 Walsh JR, McKeough ZJ, Morris NR, et al. Metabolic disease and participant age are independent predictors of response to pulmonary rehabilitation. J Cardiopulm Rehabil Prev 2013; 33: 249-256.

67 Young P, Dewse M, Fergusson W, et al. Respiratory rehabilitation in chronic obstructive pulmonary disease: predictors of nonadherence. Eur Respir J 1999; 13: 855-859.

68 Charlson M, Szatrowski TP, Peterson J, et al. Validation of a combined comorbidity index. J Clin Epidemiol 1994; 47: 1245-1251.

69 McNamara RJ, McKeough ZJ, McKenzie DK, et al. Water-based exercise in COPD with physical comorbidities: a randomised controlled trial. Eur Respir J 2013; 41: 1284-1291.

70 Tan KH, De Cossart L, Edwards PR. Exercise training and peripheral vascular disease. Br J Surg 2000; 87: 553-562.

71 Bennell KL, Hinman RS. A review of the clinical evidence for exercise in osteoarthritis of the hip and knee. $J$ Sci Med Sport 2011; 14: 4-9.

72 O'Gorman DJ, Krook A. Exercise and the treatment of diabetes and obesity. Med Clin North Am 2011; 95: 953-969.

73 Rees K, Taylor RS, Singh S, et al. Exercise based rehabilitation for heart failure. Cochrane Database Syst Rev 2004; 3: CD003331.

74 Spruit MA, Eterman RM, Hellwig VA, et al. Effects of moderate-to-high intensity resistance training in patients with chronic heart failure. Heart 2009; 95: 1399-1408.

75 Franssen FM, Wouters EF, Schols AM. The contribution of starvation, deconditioning and ageing to the observed alterations in peripheral skeletal muscle in chronic organ diseases. Clin Nutr 2002; 21: 1-14.

76 McMurray JJ, Adamopoulos S, Anker SD, et al. ESC Guidelines for the diagnosis and treatment of acute and chronic heart failure 2012: the Task Force for the Diagnosis and Treatment of Acute and Chronic Heart Failure 2012 of the European Society of Cardiology. Developed in collaboration with the Heart Failure Association (HFA) of the ESC. Eur Heart J 2012; 33: 1787-1847.

77 Thomas DE, Elliott EJ, Naughton GA. Exercise for type 2 diabetes mellitus. Cochrane Database Syst Rev 2006; 3: CD002968.

78 Marquis K, Maltais F, Duguay V, et al. The metabolic syndrome in patients with chronic obstructive pulmonary disease. J Cardiopulm Rehabil 2005; 25: 226-232.

79 Vivodtzev I, Minet C, Wuyam B, et al. Significant improvement in arterial stiffness after endurance training in patients with COPD. Chest 2010; 137: 585-592.

80 Cavalcante JL, Lima JA, Redheuil A, et al. Aortic stiffness: current understanding and future directions. J Am Coll Cardiol 2011; 57: 1511-1522.

81 Gale NS, Duckers JM, Enright S, et al. Does pulmonary rehabilitation address cardiovascular risk factors in patients with COPD? BMC Pulm Med 2011; 11: 20.

82 Griffiths TL, Burr ML, Campbell IA, et al. Results at 1 year of outpatient multidisciplinary pulmonary rehabilitation: a randomised controlled trial. Lancet 2000; 355: 362-368.

83 Güell R, Resqueti V, Sangenis M, et al. Impact of pulmonary rehabilitation on psychosocial morbidity in patients with severe COPD. Chest 2006; 129: 899-904.

84 Coventry PA, Hind D. Comprehensive pulmonary rehabilitation for anxiety and depression in adults with chronic obstructive pulmonary disease: systematic review and meta-analysis. J Psychosom Res 2007; 63: 551-565.

85 Vanfleteren LE, Franssen FM, Uszko-Lencer NH, et al. Frequency and relevance of ischemic electrocardiographic findings in patients with chronic obstructive pulmonary disease. Am J Cardiol 2011; 108: 1669-1674. 
86 Janssen DJ, Spruit MA, Leue C, et al. Symptoms of anxiety and depression in COPD patients entering pulmonary rehabilitation. Chron Respir Dis 2010; 7: 147-157.

87 Working Group on Cardiac Rehabilitation and Exercise Physiology, Working Group on Heart Failure of the European Society of Cardiology. Recommendations for exercise training in chronic heart failure patients. Eur Heart J 2001; 22: 125-135.

88 Myers J. Principles of exercise prescription for patients with chronic heart failure. Heart Fail Rev 2008; 13: 61-68.

89 Rutten FH, Cramer MJ, Grobbee DE, et al. Unrecognized heart failure in elderly patients with stable chronic obstructive pulmonary disease. Eur Heart J 2005; 26: 1887-1894. 